\section{INMIGRANTES VENEZOLANOS EN HEMODIÁLISIS ATENDIDOS EN EL MINISTERIO DE SALUD DE PERÚ}

\author{
VENEZUELAN IMMIGRANTS ON \\ HEMODIALYSIS TREATED AT THE MINISTRY \\ OF HEALTH IN PERU
}

\begin{abstract}
Vicente Aleixandre Benites-Zapata ${ }^{1, a}$, Percy Herrera-Añazco ${ }^{1, b}$, Christopher Yarasca-Zegarra ${ }^{2, c}$, Diego Venegas-Ojeda ${ }^{2, c}$, Vladimir Flores-Benites ${ }^{2, c}$
\end{abstract}

Sr. Editor. La situación política en Venezuela ha ocasionado la crisis migratoria más grande de esta parte del mundo ${ }^{(1)}$. Colombia fue el primer país en recibir a esta migración y ha significado, para su sistema de salud, problemas en el manejo de enfermedades no transmisibles como la cobertura de hemodiálisis (HD) para los pacientes con enfermedad renal crónica (ERC) ${ }^{(2)}$.

Perú es el segundo país en recibir migrantes venezolanos ${ }^{(1)} y$ es probable que, al igual que Colombia, su sistema de salud se vea desbordado por la atención de pacientes con ERC. A pesar de que el Ministerio de Salud (MINSA) ha mejorado la cobertura de HD, existen regiones del Perú donde no existen centros de diálisis ${ }^{(3)}$. En ese sentido, el objetivo del presente estudio es describir la frecuencia y características demográficas y de atención de salud de los pacientes venezolanos en HD con cobertura de atención por el Fondo Intangible Solidario en Salud (FISSAL) del MINSA.

Se realizó un estudio transversal cuya muestra corresponde a afiliados al Seguro Integral de Salud de nacionalidad venezolana atendidos en Instituciones Prestadoras de Servicios de Salud (IPRESS) privadas contratadas por el FISSAL para brindar el servicio de HD. La base de datos con información de los pacientes atendidos en IPRESS se obtuvo por comunicación directa con el FISSAL en julio del 2019. Se escogió los datos de abril pues era la información más actualizada al momento del estudio y el objetivo era dar a conocer los casos prevalentes durante ese mes. A cada paciente se le asigno un código. Se midieron variables

\footnotetext{
Universidad San Ignacio de Loyola. Unidad de Investigación para la Generación y Síntesis de Evidencias en Salud. Lima, Perú

2 Fondo Intangible Solidario de Salud. Lima, Perú

a Médico, magister en Investigación Epidemiológica; ${ }^{b}$ médico nefrólogo, magister en Docencia en Educación Superior ${ }^{\mathrm{c}}$ médico cirujano

Recibido: 02/08/2019 Aprobado: 23/10/2019 En línea:03/12/2019
}

Citar como: Benites-Zapata VA, Percy Herrera-Añazco P, Yarasca-Zegarra C, Venegas-Ojeda D, Flores-Benites V. Inmigrantes venezolanos en hemodiálisis atendidos en el Ministerio de Salud de Perú. Rev Peru Med Exp Salud Publica. 2019;36(4):709-10. doi: 10.17843/rpmesp.2019.364.4712. demográficas y características de la atención de salud. Las variables numéricas se presentaron como media y desviación estándar, previa evaluación de la normalidad con la prueba de Shapiro-Wilk. Las variables categóricas se presentaron como frecuencias absolutas.

En abril del 2019, el FISSAL financió la atención ambulatoria de HD a 21 extranjeros, 16 de los cuales eran venezolanos y cuyas características demográficas y de atención de salud, según sexo, se muestran en la Tabla 1.

Aunque la proporción de inmigrantes venezolanos es baja en relación con la población que actualmente recibe HD en el MINSA ${ }^{(4)}$, es compatible con otros reportes europeos donde los inmigrantes representan el $1,5 \%$ de la población total ${ }^{(5)}$. El bajo promedio de edad, a diferencia de lo reportado en pacientes peruanos ${ }^{(4)}$, se debe a que, 73 de cada 100 migrantes venezolanos que llegan a Perú tienen entre 20 y 39 años ${ }^{(6)}$. De igual forma, el poco tiempo en HD es debido a que el $76,8 \%$ ingresaron al Perú en el $2018{ }^{(6)}$.

En 15 de 16 pacientes, el centro de atención se ubicó en Lima, esto se explica porque el $90 \%$ de la población venezolana se ubica en esta ciudad ${ }^{(6)}$. La mayoría (11 pacientes) recibió atención en forma subsidiada y corresponde probablemente al $45 \%$ de los inmigrantes que trabajan en forma independiente y que se encontrarían en situación de vulnerabilidad; los otros cinco pacientes con cobertura independiente, probablemente correspondan al $55 \%$ de los inmigrantes que son trabajadores dependientes ${ }^{(6)}$ o tienen la posibilidad de realizar aportes mensuales al Seguro Integral de Salud.

Tabla 1. Características de la población venezolana en hemodiálisis, según sexo

\begin{tabular}{lcc}
\hline Variables & $\begin{array}{c}\text { Masculino } \\
(\mathbf{n = 8})\end{array}$ & $\begin{array}{c}\text { Femenino } \\
(\mathbf{n}=8)\end{array}$ \\
\hline Edad* $^{*}$ & $49,8(16,0)$ & $37,7(9,8)$ \\
Grupo etario (años) & & \\
$\quad<45$ & 3 & 6 \\
$\quad 45-65$ & 3 & 2 \\
$\quad>65$ & 2 & 0 \\
$\quad$ Tipo de Seguro & & \\
$\quad$ Subsidiado & 6 & 5 \\
$\quad$ Independiente & 2 & 3 \\
Lugar de Atención & & \\
$\quad$ Lima Metropolitana & 7 & 8 \\
$\quad$ La Libertad & 1 & 0 \\
Uso de Servicios de Salud Renal & & \\
$\quad$ Número de consultas por ERC* & $113(48)$ & $140(58)$ \\
$\quad$ Número de sesiones de HD* & $89(39)$ & $112(48)$ \\
$\quad$ Meses en HD* & $7,6(4,0)$ & $8,8(3,7)$ \\
\hline *Media (desviación estándar) \\
ERC: enfermedad renal crónica; HD: hemodiálisis
\end{tabular}


Aunque la baja proporción de pacientes en HD pueda parecer un problema fácilmente absorbido por el sistema de salud de Perú, como ha sido sugerido en el caso de los centros europeos ${ }^{(5)}$; es necesario recalcar que, a diferencia de Europa, el sistema de salud peruano tiene problemas de cobertura, aun en su propia población ${ }^{(3)}$.

Nuestro reporte tiene limitaciones. Primero, sólo incluye a pacientes en HD crónica y no a los que sólo reciben diálisis de emergencia. Segundo, es probable que haya pacientes que, requiriendo HD y pudiendo subsidiar su terapia, no consigan un centro de diálisis ya que residen en una región donde el MINSA no tiene cobertura ${ }^{(3)}$. Tercero, es probable que existan pacientes fallecidos en meses previos, que hayan regresado a su país o emigrado a otros. Por último, no se incluye la atención en otras instituciones prestadoras de HD.

En conclusión, la frecuencia de inmigrantes que reciben HD en el MINSA es baja, mayormente son mujeres, con terapia subsidiada y que reciben su tratamiento en Lima. Es importante considerar la necesidad de HD en esta población, como un problema emergente que podría incrementar, a corto plazo, la utilización de los servicios de salud y generar mayores costos de los que actualmente asume el FISSAL. Es probable que la cantidad de pacientes venezolanos que reciben $\mathrm{HD}$ se incremente debido a la presencia de factores de riesgo para ERC ${ }^{(6)}$. De igual forma, es probable que existan pacientes sin diagnóstico de la enfermedad pues la mayoría de los inmigrantes no acude a un centro de salud por la falta de un seguro médico ${ }^{(6)}$.

Contribución de los autores: VBZ, PHA, DVO y VFB han participado en la concepción del artículo, la recolección y análisis de datos, su redacción y aprobación de la versión final.

Conflictos de Interés: Los autores niegan conflicto de intereses.

\section{REFERENCIAS BIBLIOGRÁFICAS}

1. R4V [internet]. ACNUR 2019. Actualizado al 5 de julio del 2019. Citado el 17 de julio del 2019. Disponible en: https:// r4v.info/es/situations/platform

2. En Venezuela han muerto 5000 pacientes por falta de diálisis: exfuncionario chavista [Internet]. Miami: PanamPost; 2019 [citado el 17 de julio 2019]. Disponible en: https:// es.panampost.com/josefina-blanco/2019/03/20/dialisispacientes/

3. Herrera-Añazco P, Atamari-Anahui N, Flores-Benites V. Número de nefrólogos, servicios de hemodiálisis y tendencia de la prevalencia de enfermedad renal crónica en el Ministerio de Salud de Perú. Rev Peru Med Exp Salud Publica. 2019;36(1):627. doi: 10.17843/rpmesp.2019.361.4253.

4. Loza Munaris C, Ramos Muñoz W. Análisis de la Situación de la enfermedad renal crónica en el Perú - 2015 [Internet]. Lima: Dirección General de Epidemiología; 2015. [Revisado en julio del 2019]. Disponible en: https://www.dge.gob.pe/portal/index. php?option $=$ com_content\&view $=$ article\&id $=598 \&$ Itemid $=353$
5. Van Biesen W, Vanholder R, Ernandez T, Drewniak D, Luyckx V. Caring for Migrants and Refugees With End-Stage Kidney Disease in Europe. Am J Kidney Dis. 2018;71(5):701-9. doi: 10.1053/j.ajkd.2017.10.015.

6. Superintendencia Nacional de Migraciones. Características sociodemográficas de ciudadanos venezolanos que tramitaron su permiso temporal de permanencia en el Perú, 2017-2018 [Internet]. Lima: OGPP; 2018 [Citado el 15 de julio 2019] Disponible en : https://www.migraciones.gob.pe/estadisticas/ Informe-Estadistico-Ciudadanos-Venezolanos-2018.pdf

Correspondencia: Percy Herrera-Añazco

Dirección: Olavegoya 1879 Jesús María. Lima, Perú

Correo electrónico:pherrera@usil.edu.pe

\section{LEPTOSPIROSIS EN ARDILLAS (Sciurus stramineus) EN UNA UNIVERSIDAD DEL NORTE DE PERÚ

\author{
LEPTOSPIROSIS IN SQUIRRELS \\ (Sciurus stramineus) \\ AT A UNIVERSITY IN NORTHERN PERU
}

\author{
Raphael Sandro Gomez-Lama1,a, \\ Rosario Nelly Elera-Ojeda ${ }^{1, b}$
}

Sr. Editor. Durante «El niño costero» en el Perú se presentaron las condiciones epidemiológicas para la presentación de leptospirosis. Los departamentos norteños son los más afectados por las lluvias e inundaciones. En el departamento de Piura los distritos de Piura, Castilla y Tambo Grande fueron los más afectados ${ }^{(1)}$. Los roedores, como la ardilla nuca blanca (Sciurus stramineus), cuyas poblaciones son endémicas en la costa noroeste del Perú (2); son considerados hospederos reservorios de especies patógenas de Leptospira sp., causante de la leptospirosis. La leptopirosis es una zoonosis, de alta prevalencia en países tropicales y en vías de desarrollo ${ }^{(3)}$. Además, la falta de una vacuna que proteja contra todos los serovares

\footnotetext{
1 Universidad Nacional de Piura. Piura, Perú.

a Magister en ciencias veterinarias; ${ }^{\text {b }}$ doctora en Microbiologia

Recibido: 13/08/2019 Aprobado:30/10/2019 En línea:03/12/2019
}

Citar como: Gomez-Lama RS, Elera-Ojeda RN. Leptospirosis en ardillas (Sciurus stramineus) en una universidad del norte de Perú. Rev Peru Med Exp Salud Publica. 2019;36(4):710-2. doi: 10.17843/rpmesp.2019.364.4742. 